\title{
Activation of MEK2 is sufficient to induce skin papilloma formation in transgenic zebrafish
}

\author{
Chih-Ming Chou ${ }^{1,2}$, Yi-Chung Chen ${ }^{3}$, San Su ${ }^{3}$, Gen-Der Chen ${ }^{3}$, Kai-Yun Huang ${ }^{3}$, Huang-Wei Lien ${ }^{3}$, \\ Chang-Jen Huang ${ }^{3^{*}}$ and Chia-Hsiung Cheng ${ }^{1,2,4^{*}}$ (i)
}

\begin{abstract}
Background: Mutations in mitogen-activated protein kinase (MAPK) kinase 1 (MEK1) that occur during cell proliferation and tumor formation are well described. Information on the roles of MEK2 in these effects is still limited. We established a constitutive MEK2 transgenic zebrafish, Tg(krt14:MEK2S219D-GFP), to elucidate the role of MEK2 in skin tumor formation.

Results: We found that both constitutive MEK2 and MEK1 are able to phosphorylate the extracellular signal-regulated kinase 1 (ERK1) protein. Transient expression of constitutive MEK2 and MEK1 in the zebrafish epidermis induced papillary formation at $48 \mathrm{~h}$ post-fertilization, but no effects were observed due to the expression of MEK1, MEK2, or the dominant negative form of MEK2. The transgenic zebrafish, Tg(krt14:MEK2S219D-GFP), developed skin papillomas in the epidermis within 6 days post-fertilization (dpf). The phospho-ERK signal was detected in section of skin papillomas in an immunohistochemical experiment. Treatment with $50 \mu \mathrm{M}$ of the MEK inhibitor, U0126, had significantly decreased the skin papilloma formation in Tg(krt14:MEK2S219D-GFP) zebrafish by $6 \mathrm{dpf}$. In vitro and in vivo proliferation assay in COS-1 cells and in Tg(krt14:MEK2S219D-GFP) transgenic fish show significantly increased cell number and Ki-67 signaling.

Conclusion: Our data indicate that MEK2 is sufficient to induce epidermal papilloma formation through MAPK signaling in zebrafish, and this transgenic model can be used as a new platform for drug screening.
\end{abstract}

Keywords: MEK2, Skin, Papilloma, Proliferation, Zebrafish

\section{Background}

The Raf/mitogen-activated protein kinase (MAPK) kinase (MEK)/extracellular signal-regulated kinase (ERK) cascade is the downstream pathway of Ras which transforms its effects on its targets [15]. When Ras is activated by a mutation, it was hypothesized that downstream components will be constitutively activated and corresponding abnormal gene expressions will occur that drive proliferation. Several model systems of skin tumors in mice have been established $[1-5,7,8,12,20]$. Zebrafish are a very useful and convenient model organism for studying human diseases. So far, no squamous skin cell tumor model has been established in zebrafish $[9,10,16,25,29]$.

MEK2 was reported to be a factor related to invasion and metastasis of pancreatic tumors [28]. The expression

\footnotetext{
* Correspondence: cjibc@gate.sinica.edu.tw; chcheng@tmu.edu.tw ${ }^{3}$ Institute of Biological Chemistry, Academia Sinica, 128 Academia Rd., Sec 2, Taipei 115, Taiwan

'Department of Biochemistry and Molecular Cell Biology, School of Medicine, College of Medicine, Taipei Medical University, Taipei 110, Taiwan Full list of author information is available at the end of the article
}

of Raf22w, v-Ha-Ras, or v-Src resulted in the constitutive activation of MEK1 and MEK2 [11]. A mutation of p21ras in hepatocellular carcinoma (HCC) was associated with marked changes in expression of the function of MAPK cascades mainly through MEK1 [19]. MEK1 is important for skin tumor development, and MEK2 could not compensate for this process with the loss of MEK1 function in a mouse model [27]. The MEK1 and MEK2 signaling pathways were not redundant or interchangeable for cell proliferation in SK-MEL-28 melanoma cells [17].

However, it is unclear if MEK2 constitutively activated in zebrafish is sufficient to induce epidermal neoplasia. Herein, we established a stable transgenic zebrafish model with consistent expression of MEK2 ${ }^{\text {S219D }}$ under an epidermal-specific Krt14 promoter using the Tol2transposone system to investigate the effects of MEK2 $^{\text {S219D }}$ on inducing epidermal neoplasias in vivo. Further treatment with U0126 in this transgenic zebrafish tumor model was used to evaluate the cause of skin papilloma. We suggest that this model can serve as a useful platform for cancer drug screening. 


\section{Methods}

Fish

Zebrafish (Danio rerio) were maintained at $28^{\circ} \mathrm{C}$ on a 14 h light/10-h dark cycle. Embryos were incubated at $28{ }^{\circ} \mathrm{C}$, and different developmental stages were determined according to the description in the Zebrafish Book [30].

\section{Total RNA isolation}

Total RNA was isolated from fertilized eggs (a pool of embryos at $12,24,36,48,72,96,120$, and 144 h postfertilization; hpf), using the RNAzol reagent (Tel-Test, Friendswood, TX, USA) according to instructions of the manufacturer.

\section{Isolation of the full-length mek1, mek2, and erk1 cDNAs of zebrafish}

cDNAs encoding the complete open reading frame (ORF) of zebrafish mek1/2 and erk 1 were obtained by polymerase chain reaction (PCR) amplification using gene-specific primers according to the NCBI's zebrafish EST database. PCR amplification was performed in a 50$\mu \mathrm{l}$ reaction mixture containing $2 \mu \mathrm{l}$ of first-strand $\mathrm{cDNA}$, $0.5 \mu \mathrm{g}$ of a forward primer (MEK1-F: 5'-CGG GAT CCA TGC AGA AAA GGA GGA AG-3'; MEK2-F: 5' TAT TGG ATG TCA GTA GAG ACA ACC TGG-3'; and ERK1-F: 5' -CGG ACA GAA ACG ATG GCG GAA TCG-3') and reverse primer (MEK1-R: 5'-GGG AAT TCC ATT CCC ACA CTG TGA GT-3'; MEK2-R: 5'TTC AGC GCT ATG AGT GGG TGT GCT AGG-3'; and ERK1-R: 5 '-CGT GAT GAC TGT CCC TCT CAG GAG-3'), $1.5 \mathrm{mM} \mathrm{MgCl}_{2}, 0.2 \mathrm{mM}$ dNTP, and 2.5 units ExTaq (Takara Shuzo, Shiga, Japan). Samples were incubated in a thermal cycler (SensoQuest, Göttingen, Germany). PCR amplification was performed; the DNA fragment of MEK1 and MEK2 was cloned into a pGEM$\mathrm{T}$ Easy vector (Promega, Madison, WI, USA) as pGEMT-MEK1 and pGMET-MEK2 plasmids, and the resultant clones were subjected to a sequencing analysis.

\section{Site-directed mutagenesis of zebrafish MEK1 and MEK2 cDNA}

Site-directed mutagenesis was performed to generate plasmids encoding MEK1/2 mutants (S219D and S223A) using the pGEMT-MEK1 and pGEMT-MEK2 plasmid as a template for PCR amplification using specific oligonucleotide primers. The corresponding oligonucleotides used for PCR reaction were as follows with the altered bases underlined: MEK1S219D-F: 5'-GGA CAA CTC ATT GAC GAC ATG GCC-3', MEK1S219D-R: 5' -GGC CAT GTC GTC AAT GAG TTG TCC-3'; MEK2S219DF: 5' -CAG CTC ATC GAC GAT ATG GCC AAC TCC3', MEK2S219D-R: 5'-GGA GTT GGC CAT ATC GTC GAT GAG CTG-3'; and MEK2S223A-F: 5'-ATG GCC AAC GCC TTC GTT GGA ACA CGG-3', MEK2S223A-
R: $5^{\prime}$-CCG TGT TCC AAC GAA GGC GTT GGC CAT3 '. Sequences of the resultant plasmids were verified using DNA sequencing.

\section{Construction of the expression plasmids}

The 4-kb upstream region of the zebrafish krt14 gene (krt14 promoter) was amplified from a BAC clone, DKEYP-113D7 (Source Biosicence, Nottingham, UK), and inserted into the pGEM-T Easy cloning vector (Promega, Madison, WI, USA) as pGMET-krt14-pro plasmid. The krt14 promoter was then inserted into the pTol2-GFP vector [14] at appropriate sites to generate the pTol2-krt14-GFP plasmid. MEK1, MEK1S219D, MEK2, MEK2S219D, MEK2S223A, and ERK1 were inserted into pcDNA3-mCherry, pcDNA3-GFP, and pcDNA3-HA from PCR products as pcDNA3-MEK1mCherry, pcDNA3-MEK1S219D-GFP, pcDNA3-MEK2GFP, pcDNA3-MEK2S219D-GFP, pcDNA3-MEK2S223AGFP, pcDNA3-ERK1-HA and then inserted into the pTol2krt14-GFP vector to generate pTol2-krt14-MEK1-mCherry, pTol2-krt14-MEK1S219D-GFP, pTol2-krt14-MEK2-GFP, pTol2-MEK2S223A-GFP, and pTol2-krt14-MEK2S219DGFP plasmids. The pcDNA3-GFP and pcDNA3-mCherry plasmids were constructed by inserting cDNA corresponding to the green fluorescence protein (GFP) and mCherry coding-region into pCMV-HA-YUN, which was a gift from Dr. H.J. Kung (Davis Cancer Center, University of California, Sacramento, CA, USA).

Microinjection of expression plasmid into zebrafish embryo The expression plasmid was adjusted to a final concentration of $100 \mu \mathrm{g} / \mathrm{ml}$ in $1 \times$ Danieau solution (5 mM HEPES at pH 7.6, $58 \mathrm{mM} \mathrm{NaCl}, 0.7 \mathrm{mM} \mathrm{KCl}, 0.4 \mathrm{mM}$ $\mathrm{MgSO}_{4}$, and $\left.0.6 \mathrm{mM} \mathrm{Ca}\left(\mathrm{NO}_{3}\right)_{2}\right)$ containing $0.5 \%$ phenol red and injected into a zebrafish embryo in the one-cell stage using the Narishige IM 300 microinjector system (Narishigi Scientific Instrument, Tokyo, Japan).

\section{Cell culture and plasmid transfection}

Monkey kidney fibroblast COS-1 cells (ATCC CRL-1650; VA, USA) were cultured in high-glucose Dulbecco's modified Eagle's medium (DMEM; Hyclone, UT, USA), supplemented with $10 \%$ fetal bovine serum (FBS; Hyclone, UT, USA) in a humidified atmosphere of $5 \% \mathrm{CO}_{2}$ at $37{ }^{\circ} \mathrm{C}$. COS-1 cells were transfected with pcDNA3, pcDNA3MEK1-mCherry, pcDNA3-MEK1S219D-GFP, pcDNA3MEK2-GFP, pcDNA3-MEK2S219D-GFP, pcDNA3-ERK1HA plasmids, using Lipofectamine Plus reagent (Life technologies, Grand Island, NY, USA) according to manufacturer's manner. Culture cells were harvested at $48 \mathrm{~h}$ post-transfection and lysed with lysis buffer. The protein level was analyzed by western blotting. 


\section{Immunoblot analysis}

Total cell lysates from transfected cells were harvested and separated on $10 \%$ sodium dodecylsulfate (SDS)-polyacrylamide gels and transferred to polyvinylidene difluoride (PVDF) membranes. Membranes were blocked with $5 \%$ skim milk in phosphate-buffered saline (PBS) for $1 \mathrm{~h}$ at room temperature and then incubated at $4{ }^{\circ} \mathrm{C}$ with an antiHA monoclonal antibody, anti-Actin polyclonal antibody (Santa Cruz, Dallas, TX, USA), and anti-mCherry polyclonal antibody (GeneTex, Hsinchu, Taiwan). Signals were detected using Immobilon Western Chemiluminescent HRP Substrate (Millipore, Billerica, MA, USA).

\section{Subcellular localization and image analysis}

COS-1 cells transfected with pcDNA3-ERK1-HA alone or with pcDNA3-MEK1-mCherry, pcDNA3-MEK1S219DGFP, pcDNA3-MEK2-GFP, pcDNA3-MEK2S219D-GFP, and pcDNA3-MEK2S223A-GFP for $48 \mathrm{~h}$ were fixed with 4 $\%$ paraformaldehyde and permeabilized in PBS with $0.1 \%$ Triton X-100. Immunostaining was performed using an anti-HA antibody at $4{ }^{\circ} \mathrm{C}$ overnight, followed by incubation with a Cy2-conjugated goat anti-mouse antibody or Cy3conjugated goat anti-mouse antibody for $30 \mathrm{~min}$ and DAPI $(1 \mu \mathrm{g} / \mathrm{ml}$ ) for $5 \mathrm{~min}$ at room temperature. Photoimages were prepared using an Olympus IX70-FLA inverted fluorescence microscope (Olympus, Tokyo, Japan) equipped with the SPOT system (Diagnostic Instruments, Sterling Heights, MI, USA).

\section{Histology and Immunohistochemistry}

Larvae in which a skin papilloma had formed from $\operatorname{Tg}($ krt14-MEK2S219D-GFP) at 6 days post-fertilization (dpf) were fixed with $4 \%$ paraformaldehyde for 1 day and dehydrated with $100 \%$ methanol. Specimens were embedded in paraffin, sliced and stained with hematoxylin and eosin (H\&E) or incubated with $3 \%$ serum at room temperature for $1 \mathrm{~h}$ before incubation with an anti-pERK polyclonal antibody (Santa Cruz, Dallas, TX, USA) or anti-Ki-67 polyclonal antibody (GeneTex, Hsinchu, Taiwan) containing $5 \%$ serum and $2 \mathrm{mg} / \mathrm{ml}$ bovine serum albumin (BSA) at $4{ }^{\circ} \mathrm{C}$ overnight. After washing with PBST, embryos were incubated with a horseradish peroxidase (HRP)-conjugated goat anti-rabbit secondary antibody at room temperature for $3 \mathrm{~h}$. HRP labeling was visualized using DAB (Merck, Darmstadt, Germany) as a substrate. Nuclei were counterstained with hematoxylin (Merck, Darmstadt, Germany).

\section{Chemical treatment and statistical analysis}

$\mathrm{Tg}(\mathrm{krt14}-\mathrm{GFP})$ and $\mathrm{Tg}(\mathrm{krt14}-\mathrm{MEK} 2 \mathrm{~S} 219 \mathrm{D}-\mathrm{GFP})$ embryos were dechorionated at $1 \mathrm{dpf}$. Ten embryos with high GFP fluorescent signaling were transferred to each well in $1 \mathrm{ml}$ water containing $50 \mu \mathrm{M}$ U0126 (Sigma, MO, USA) or $0.01 \%$ DMSO (Sigma, MO, USA) and incubated from 1 to 6 days at $28{ }^{\circ} \mathrm{C}$ incubator. The number of zebrafish forms any papilloma was assessed at $6 \mathrm{dpf}$ under an Olympus IX70-FLA inverted fluorescence microscope. Results from three independent experiments are expressed as the mean \pm standard deviation (SD). Statistical significance between groups was determined by an unpaired Student's t-test. A probability of $p<0.001$ was indicated as $* *$.

\section{Cell proliferation assay}

Cell proliferation was performed by trypan blue assay. Briefly, $3 \times 10^{4}$ COS-1 cells were seeded in 24-well plate overnight. COS-1 cells were transfected with $1 \mu \mathrm{g}$ pcDNA3-GPF or pcDNA3-MEK2S219D-GFP plasmids using Lipofectamine Plus reagent (Life technologies, Grand Island, NY, USA) according to manufacturer's manner. Culture cells were trypsinized and the number of viable cells was virtually counted with a hemocytometer at day 1 , 2, 3 and 4 post-transfection. Three counts per well were performed on three different experiments. Statistical significance between groups was determined by an unpaired Student's t-test. A probability of $p<0.05$ was indicated as *.

\section{Results \\ Cloning and characterization of the zebrafish mek 1 and mek2 (mek2a) cDNAs}

The MEK family has 7 members, MEK1 MEK7, in mammals. According to genomic predictions from NCBI zebrafish EST/cDNA, we identified 7 mek genes (mek1, mek2, mek3, mek4a, mek4b, mek5, and mek7) in zebrafish. Phylogenetic analysis of these 7 MEK proteins with mammalian counterparts indicated that only MEK3 but not MEK6 was found in zebrafish; instead, there is a duplication of MEK4 to form MEK4A and MEK4B. Each member of the MEK family was separated into individual monophyletic groups (Fig. 1a).

The mek 1 and mek2 cDNAs were isolated from mixed cDNA pools prepared from different developmental stages of zebrafish embryos by PCR amplification. Fulllength cDNA of zebrafish mek1 and mek2 were 1191 and 1194 base-pair long, encoding two proteins of 396 and 397 amino acid residues, respectively. The overall amino acid sequence of zebrafish MEK1 showed $76 \%$ identity and $85 \%$ similarity to that of MEK2. The zebrafish MEK1 respectively showed 88 and $87 \%$ identities to human and mouse MEK1 and shown $78 \%$ identities to human and mouse MEK2 at the level of amino acid sequences (Fig. 1b). The encoded amino acid sequence of zebrafish MEK1 and MEK2 was highly homologous to human and mouse proteins. The phosphorylated site of MEK1 was also conserved (Fig. 1b, arrow). These data suggest that zebrafish MEK1 and MEK2 are highly conserved with mammalian MEK1 and MEK2. 


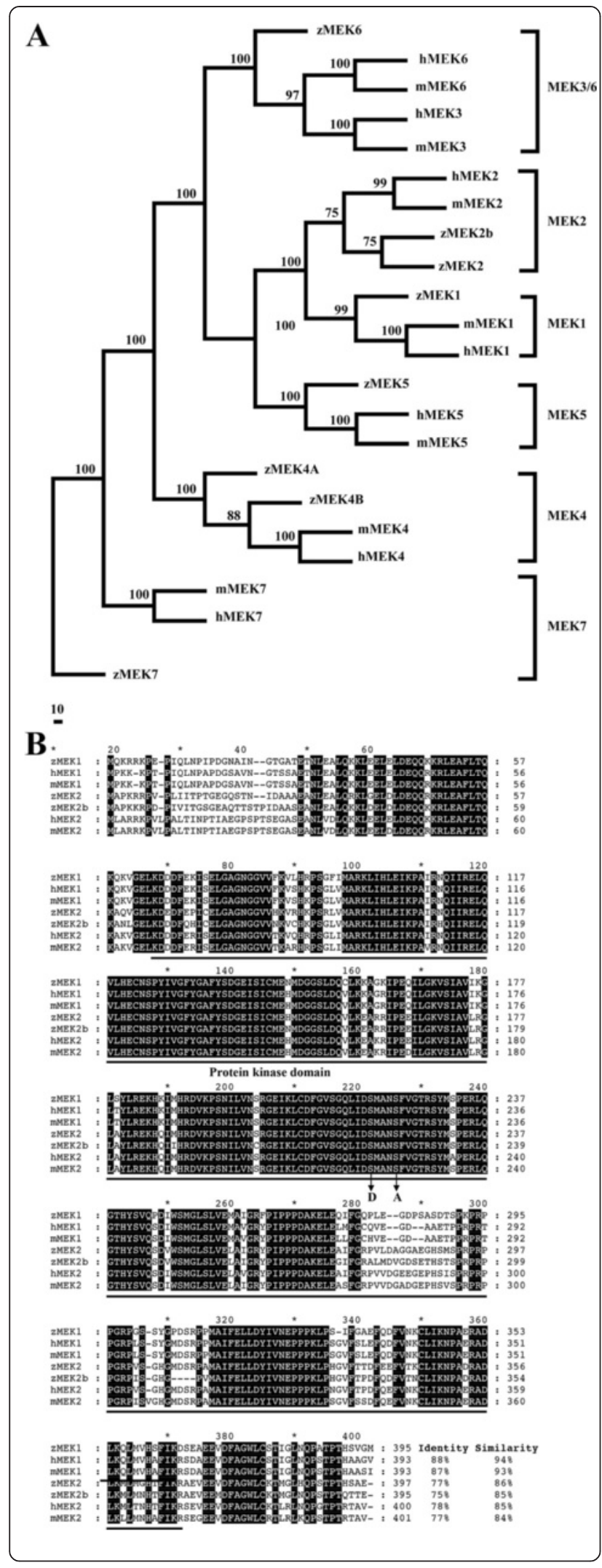

Fig. 1 a. Phylogenetic analysis of the MEK gene family in the human, mouse, and zebrafish. Each member of the MEK family was separated into individual monophyletic groups. Amino acid sequences were aligned with ClustalW. Accession numbers for these sequences are as follows: ZMEK1 (NP_998584), hMEK1 (NP_002746), mMEK1 (NP_032953), zMEK2 (zMEK2a, NP_001032468), zMEK2b (NP_001121753), hMEK2 (NP_109587), mMEK2 (NP_075627), hMEK3 (NP_659731), mMEK3 (NP_032954), zMEK4A (NP_991299), zMEK4B (NP_001082890), hMEK4 (AAH60764), mMEK4 (NP_033183), zMEK5 (NP_001107789), hMEK5 (AAA96146), mMEK5 (BAA82040), ZMEK6 (NP_571799), hMEK6 (AAB05035), mMEK6 (AAH75652), zMEK7 (XP_689405), hMEK7 (O14733), mMEK7 (Q8CE90). b. Protein sequence alignment of zebrafish MEK1 and MEK2 with those of the human and mouse. The predicted protein kinase domain is indicated. The amino acid sequence of zebrafish MEK1 showed high conservation with human (88\% identity and $94 \%$ similarity) and mouse MEK1. Zebrafish MEK1 shared $76 \%$ identity and $85 \%$ similarity in the protein sequence with MEK2. Mutation sites of the constitutively activated (S219D) and dominant negative (S223A) forms of zebrafish MEK1 and MEK2 are indicated. Accession numbers for these sequences are as follows: ZMEK1 (NP_998584), hMEK1 (NP_002746), mMEK1 (NP_032953), ZMEK2 (ZMEK2a, NP_001032468), zMEK2b (NP_001121753), hMEK2 (NP_109587), mMEK2 (NP_075627)

Both MEK1 and MEK2 phosphorylated the ERK1 protein In the MAPK signaling pathway, ERK proteins are phosphorylated by activated MEK1 and MEK2 to induce downstream gene transcription. Phosphorylation at serine 218 and 222 at human MEK1 by the Raf protein activated ERK1/2 phosphorylation. To elucidate the phosphorylation activity of zebrafish MEK1 and MEK2, we identified and cloned the $m e k 1$, mek2, and erk 1 cDNAs from zebrafish embryonic cDNA. The human MEK1 phosphorylation sites of serine 218 and 222 were identified as serine 219 and 223 of zebrafish MEK1 and MEK2 (Fig. 1b). Sitedirected mutations of S219D and S223A were performed at zebrafish MEK1 and MEK2 as MEK1 ${ }^{\text {S219D }}$, MEK2 ${ }^{\text {S219D }}$, and MEK2 ${ }^{\text {S223A }}$. Expression plasmid constructs of zebrafish MEK1, MEK1 ${ }^{\text {S219D }}$, MEK2, MEK2 ${ }^{\text {S219D }}$, MEK2 ${ }^{\text {S223A }}$, and ERK1 were constructed into the pcDNA3-mCherry, pcDNA3-GFP, and pcDNA3-HA plasmids to produce pcDNA3-MEK1-mCherry, pcDNA3-MEK1S219D-GFP, pcDNA3-MEK2-GFP, pcDNA3-MEK2S219D-GFP, pcDNA3MEK2 S223A, and pcDNA3-ERK1-HA, respectively. Transfection of the pcDNA3-ERK1-HA plasmid individually or with the pcDNA3-MEK1-mCherry, pcDNA3-MEK1S219D-GFP, pcDNA3-MEK2-GFP, or pcDNA3-MEK2S219D-GFP plasmid into COS-1 cells was used to detect phosphorylation of ERK1. A Western blot analysis showed that MEK1 and MEK2 were unable to phosphorylate ERK1. In contrast, constitutively activated MEK1 $^{\text {S219D }}$ and MEK2 ${ }^{\text {S219D }}$ could phosphorylate ERK1 (Fig. 2a). These data suggest that zebrafish MEK1 and MEK2 both exhibit ability to phosphorylate ERK1.

It has been shown recently that overexpression of the ERK protein without active signaling caused its accumulation in the nuclei and the cytoplasm. In the presence of the active MEK protein, the ERK protein was 


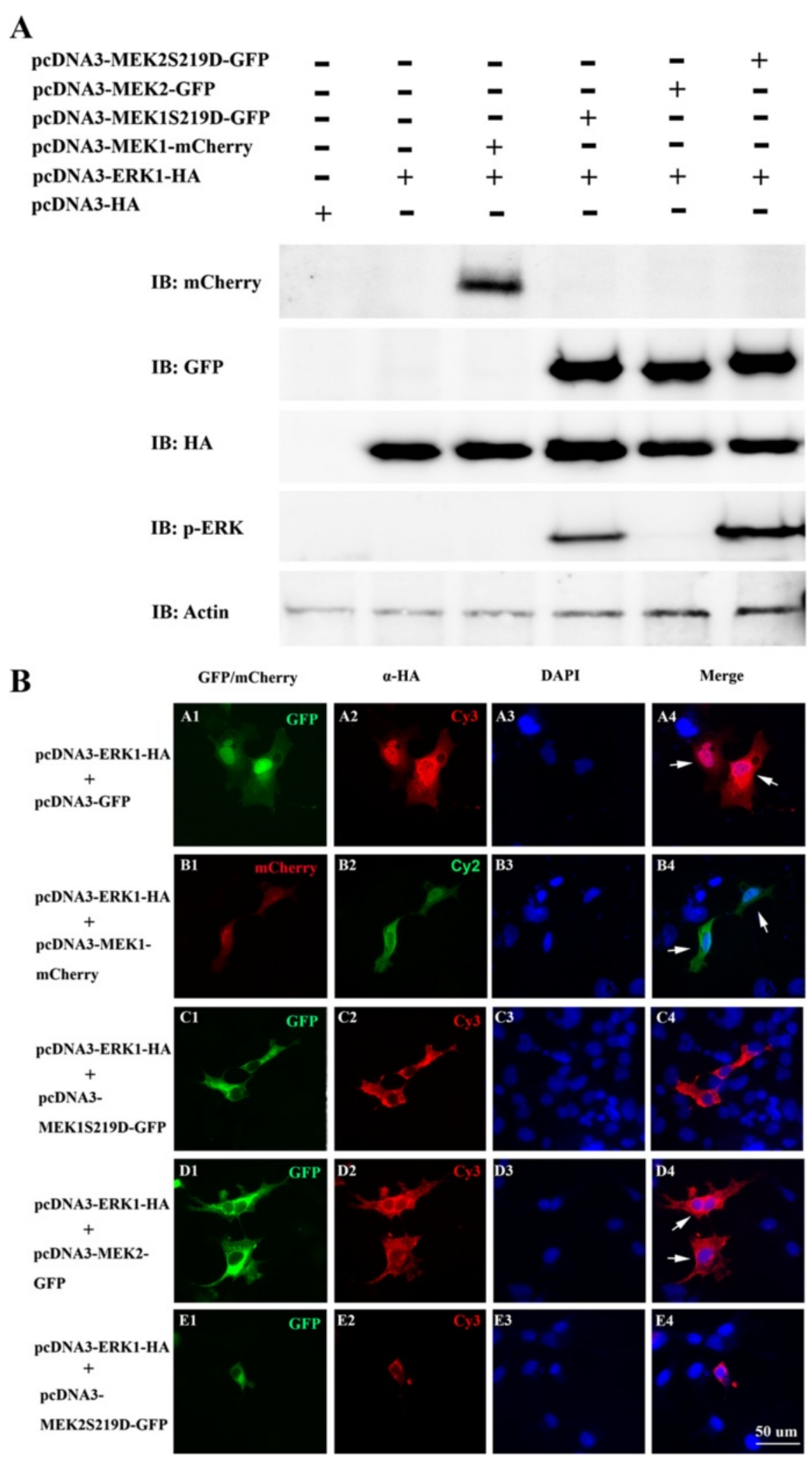

Fig. 2 (See legend on next page.) 
(See figure on previous page.)

Fig. 2 Constitutively activated MEK1 (MEK1 ${ }^{\text {S219D }}$ ) and MEK2 (MEK2 ${ }^{\mathrm{S} 219 \mathrm{D})}$ ) both phosphorylated ERK1. a. COS-1 cells were transiently transfected with pcDNA3-HA, pcDNA3-ERK1-HA, pcDNA3-MEK1-mCherry, pcDNA3-MEK1S219D-GFP, pcDNA3-MEK2-GFP, or pcDNA3-MEK2S219D-GFP. Total lysates were analyzed by Western blotting using anti-HA, anti-GFP, anti-pERK monoclonal antibodies; anti-mCherry and anti-Actin polyclonal antibodies. b. Intracellular localization of ERK1, MEK1, and MEK2 in COS-1 cells by fluorescent microscopy. The pcDNA3-ERK1-HA was co-transfected with pcDNA3-GFP (A1-A4), pcDNA3-MEK1-mCherry (B1-B4), pcDNA3-MEK1S219D-GFP (C1-C4), pCDNA3-MEK2-GFP (D1-D4), or pcDNA3-MEK2S219D-GFP (E1-E4). Cy2 or Cy3 dye used an anti-HA monoclonal antibody to detect localization of ERK1 as visualized. DAPI was used to stain nuclear DNA. White arrows indicate the ERK1 protein localized in nuclei and the cytoplasm. IB, immunoblot

translocate from nuclei into the cytoplasm [18]. To further characterize the functions of MEK1 and MEK2, we examined the cellular localization of ERK1. In GFP, MEK1 or MEK2 expressed COS-1 cells, the ERK1 protein was accumulated in the nuclei and cytoplasm (Fig. 2b. A1-A4, B1-B4, D1-D4; arrow). In the presence of active MEK $1^{\mathrm{S} 219 \mathrm{D}}$-GFP and MEK2 ${ }^{\mathrm{S} 219 \mathrm{D}}$-GFP, the ERK1 protein was located in the cytoplasm (Fig. 2b, C1-C4, E1-E4). These results indicate that active zebrafish MEK1 and MEK2 both are able to induce ERK1 protein translocate from nuclei to the cytoplasm.

\section{Transient expression of MEK $1^{\mathrm{S} 219 \mathrm{D}}$ and MEK2 ${ }^{\mathrm{S219D}}$ induced papillary formation in the zebrafish epidermis}

It was reported that constitutive activation of MEK1 could transform normal intestinal epithelial cells into tumors [7]. MEK1 and MEK2 were reported to be functionally redundant in epidermal development in mouse models [26]. MEK1 is important in skin tumor development, and MEK2 cannot compensate for loss of MEK1 function [27]. Another report indicated that MEK2 is sufficient for melanoma cell proliferation, but MEK1 is not [17]. To study the role of zebrafish MEK2 in the formation of skin tumors and comparison of expression with MEK1, pTol2-krt14-MEK2GFP, pTol2-MEK2S219D-GFP, pTol2-MEK2S223AGFP, pTol2-MEK1-mCherry and pTol2-MEK1S219DGFP were microinjected into one-cell stage of zebrafish embryos and revealed the expression at $3 \mathrm{dpf}$. The zebrafish embryos injected with pTol2-krt14MEK2-GFP (Fig. 3b), pTol2-Krt14-MEK2S223A-GFP (Fig. 3c), and pTol2-krt14-MEK1-mCherry (Fig. 3e) displayed specific GFP or mCherry expression in the skin cells (Fig. 3a). The pTol2-krt14-MEK2S219DGFP-injected embryos also displayed specific expression in the skin cells. Furthermore, several skin cells in the upper epidermis were shown to have papillae and to have budded (Fig. 3d, arrows). The pTol2krt14-MEK1S219D-GFP transgenic embryos also displayed papillae and budded skin cell formation in the epidermis (Fig. 3f). These results suggest that active zebrafish MEK2 and MEK1could promote the proliferation of skin cells to form papillae.

\section{MEK2 is sufficient to induce skin papilloma formation in transgenic zebrafish}

To further elucidate the tumorigenesis of MEK2 in zebrafish, we established a transgenic zebrafish known as $\mathrm{Tg}(\mathrm{krt14}$ :MEK2S219D-GFP) in which expression of the MEK2S219D-GFP fusion protein was driven by the krt14 promoter to establish a skin tumor animal model. The skin papilloma formation of $\mathrm{Tg}(\mathrm{krt14}$ :MEK2S219DGFP) was presented approximately $34 \%$ in homozygous and $5 \%$ in heterozygous. $\operatorname{Tg}(\operatorname{krt14}:$ MEK2S219D-GFP) zebrafish expressed the GFP signal from embryos to adult. $\operatorname{Tg}(\mathrm{krt14}$ :MEK2S219D-GFP) zebrafish exhibited skin papilla from $3 \mathrm{dpf}$ (Fig. 4b-B') and then developed to a skin tumor at $6 \mathrm{dpf}$ (Fig. 4c-C', d-D', e-E'; white-dots area). In addition, we established a transgenic line known as $\operatorname{Tg}(\mathrm{krt14}: \mathrm{GFP})$, in which GFP expression was driven by the krt14 promoter to reveal the expression pattern of skin cells. Homozygous $\mathrm{Tg}(\mathrm{krt14}$ :GFP) transgenic zebrafish exhibited the GFP signal in the entire skin cells, which was a normal phenomenon from embryos to adult (Fig. 4a-A'). These results indicated that constitutive MEK2 was sufficient to induce skin papilloma formation.

To further examine whether skin papilloma formation induced by MEK2 is through downstream signaling activation, we tested ERK phosphorylation of skin papilloma by an IHC experiment. Phosphorylated ERK is well known as a proliferation marker during tumor formation. Phosphorylation of ERK in skin papilloma of Homozygous $\operatorname{Tg}(\mathrm{krt14}$ :MEK2S219D-GFP) larvae was detected by IHC experiment (Fig. 4f-F'). These results suggested that zebrafish constitutive activated MEK2 ${ }^{\text {S219D }}$ induced skin papilloma formation through phosphorylation of downstream ERK signaling.

\section{U0126 inhibits skin papilloma formation induced by MEK2S219D}

We further examined whether skin papilloma formation in $\operatorname{Tg}(\mathrm{krt14}: M E K 2 S 219 D-G F P)$ zebrafish was induced by MEK2 activation by specific MEK1/2 inhibitor, U0126. $\mathrm{Tg}(\mathrm{krt14}$ :MEK2S219D-GFP) transgenic zebrafish were incubated in $50 \mu \mathrm{M}$ U0126 or $0.01 \%$ DMSO (Control) from 1 to $6 \mathrm{dpf}$. No significantly abnormal phenotype of $\mathrm{Tg}(\mathrm{Krt14}: \mathrm{GFP})$ zebrafish larvae was observed by treated with U0126 at 2 and 6 dpf (Fig. 5a, b). No skin papilloma 

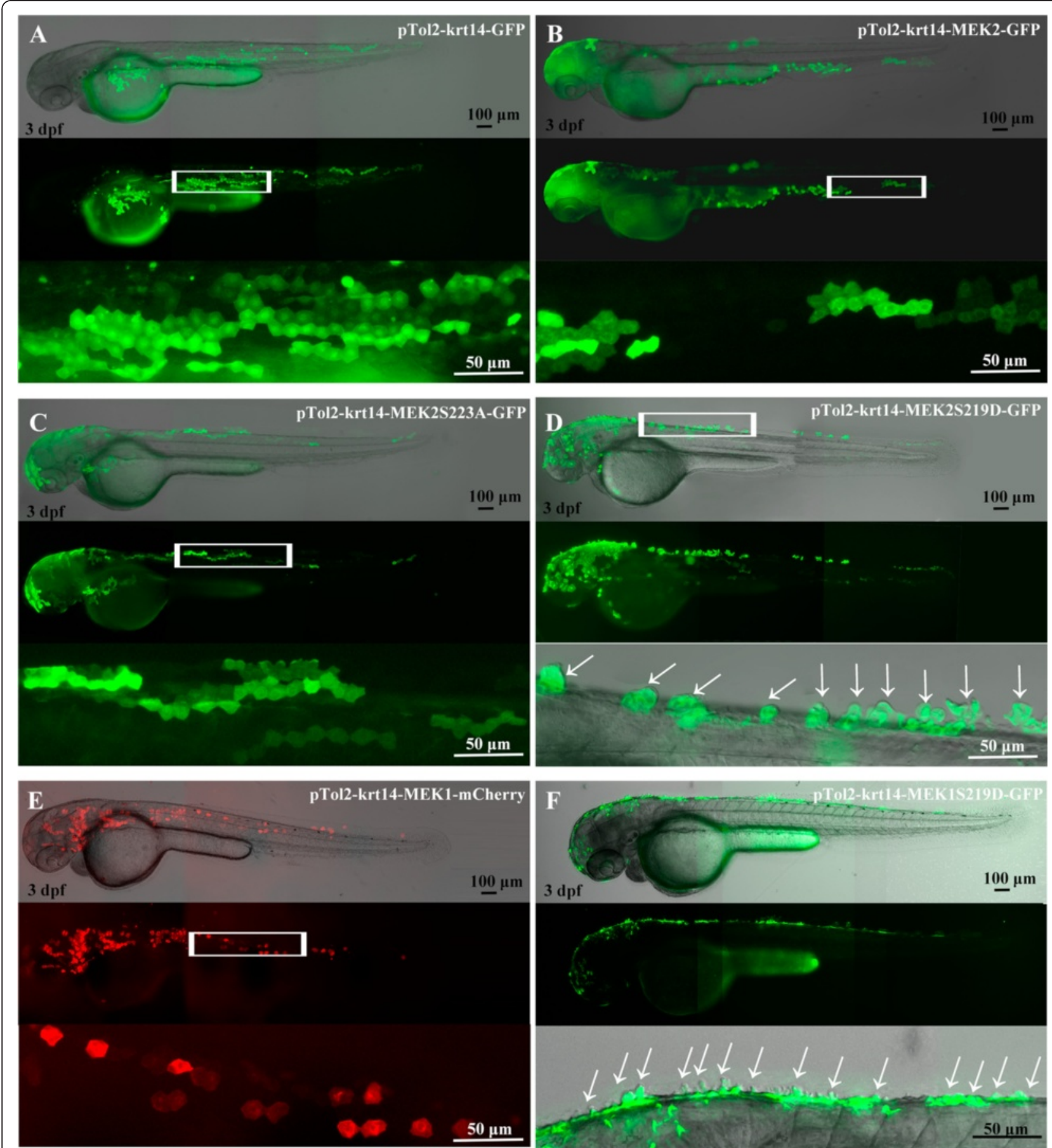

Fig. 3 Transient expressions of MEK1 and MEK2 driven by the krt14 promoter induced papillae formation in skin cells. Lateral view of pTol2-krt14-GFP (a), pTol2-krt14-MEK2-GFP (b), pTol2-MEK2S223A-GFP (c), pTol2-krt14-MEK2S219D-GFP (d), pTol2-krt14-MEK1-mCherry (e), and pTol2-krt14-MEK1S219D-GFP (f) plasmids, which were microinjected into 1-cell stage of zebrafish embryos and visualized at 3 days post-fertilization (dpf). The arrow indicates skin cell papillae and budding in the upper epidermis. The upper panel is a fluorescent image. The middle panel is merged fluorescent and bright-field images. The lower panel is an enlargement of the white box in the middle panel

was observed at $2 \mathrm{dpf}$ in $\mathrm{Tg}(\mathrm{krt14}: \mathrm{MEK} 2 \mathrm{~S} 219 \mathrm{D}-\mathrm{GFP})$ zebrafish larvae (Fig. 5c). Incubated $\mathrm{Tg}(\mathrm{krt14}$ :MEK2S219DGFP) zebrafish larvae with $50 \mu \mathrm{M}$ at $2 \mathrm{dpf}$ or $0.01 \%$ DMSO as control, no skin papilloma was observed in
U0126 treatment at $6 \mathrm{dpf}$ (Fig. 5d) but several skin papilloma was observed in DMSO treatment at $6 \mathrm{dpf}$ (Fig. 5e). The skin papilloma formation in transgenic zebrafish larvae was presented $90 \%$ in DMSO treatment and $10 \%$ 

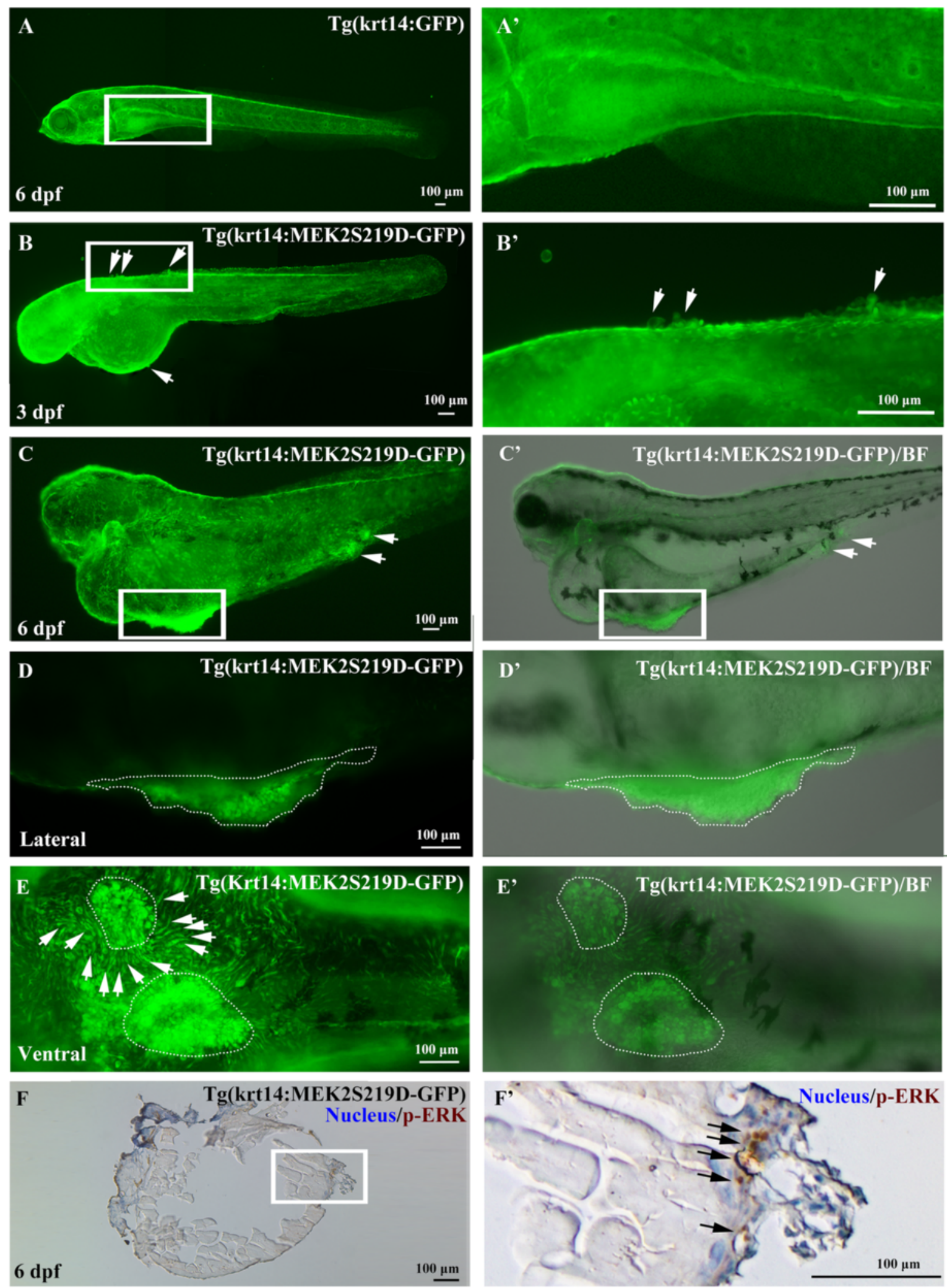

Fig. 4 (See legend on next page.) 
(See figure on previous page.)

Fig. 4 Skin tumor formation in Tg(krt14: MEK2S219D-GFP) zebrafish. Skin cells proliferated and formed papilla from 3dfp (b, B', white arrow) on the yolk skin in Tg(krt14: MEK2S219D-GFP) embryos to form a skin tumor at $6 \mathrm{dpf}$ (c, and $C_{\text {; }}$ lateral view, d and $D^{\prime}$, white-dots area; ventral view, e and $E^{\prime}$, white-dots area). Tg(krt14:GFP) embryos had developed normal skin cells at $6 \mathrm{dpf}$ (a, $\left.A^{\prime}\right)$. An immunohistochemical experiment was used to detect ERK phosphorylation in skin tumors. p-ERK was detected by a p-ERK monoclonal antibody and visualized by DAB (brown). Nuclei were counterstained with hematoxylin (blue) $\left(\mathbf{f}, F^{\prime}\right)$

in U0126 treatment at $6 \mathrm{dpf}$ (Fig. 5f). These results suggest that the skin papilloma formation in $\operatorname{Tg}(\mathrm{krt14}$ : MEK2S219D-GFP) zebrafish larvae can be inhibited by the specific MEK inhibitor, U0126. The MEK2S219D is sufficient to induce skin papilloma formation through activation of MAPK pathway.
In vitro and in vivo cell proliferation assay in COS- 1 cells and Tg(krt14:MEK2S219D-GFP) transgenic zebrafish

To further assess the effect of proliferation induced by MEK2S219D, we performed the in vitro cell proliferation and in vivo proliferation assay in $\mathrm{Tg}(\mathrm{krt14}$ :MEK2S219DGFP) transgenic zebrafish. COS-1 cells was seeded to
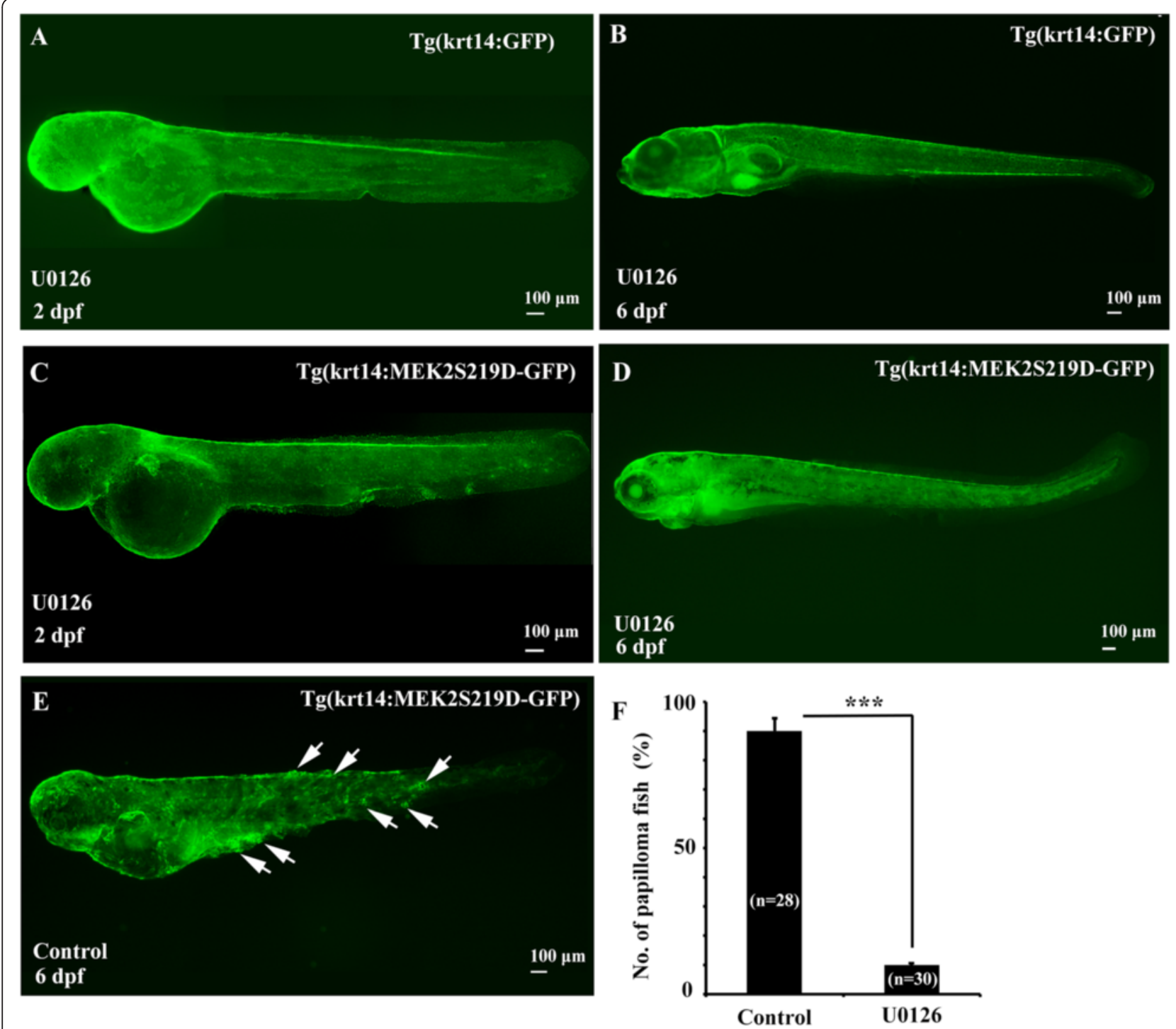

Fig. 5 The MEK inhibitor, U0126, inhibited skin tumor formation in Tg(krt14: MEK2S219D-GFP) embryos. Tg(Ka14:GFP) embryos were observed by treated with $50 \mu \mathrm{M}$ U0126 at $2 \mathrm{dpf}(\mathbf{a})$ and $6 \mathrm{dpf}(\mathbf{b})$. Tg(krt14: MEK2S219D-GFP) embryos were observed by treated with $50 \mu \mathrm{M} \cup 0126$ at $2 \mathrm{dpf}$ (c) and $6 \mathrm{dpf}$ (d) or treated with DMSO at $6 \mathrm{dpf}(\mathbf{e})$. The white arrow indicated skin papilloma. $\mathbf{f}$ The frequency of tumor formation in $\mathrm{Tg}(\mathrm{krt} 14$ : MEK2S219D-GFP) embryos incubated with DMSO (Control) or U0126 
24-well for $24 \mathrm{~h}$ and then transfected with pcDNA3GFP or pcDNA3-MEK2S219D-GFP plasmid. The cell number of transfected COS-1 cells was counted using trypan blue assay at day 1 to day 4 . The cell number of transfected with pcDNA3-MEK2S219D-GFP was found significantly higher than transfected with pcDNA3-GFP (Control) at 3 and 4 day posttransfection (Fig. 6a). The in vivo proliferation assay was performed using $\mathrm{H} \& \mathrm{E}$ and $\mathrm{IHC}$ staining in $\mathrm{Tg}(\mathrm{krt14}$ :MEK2S219D-GFP) transgenic zbrafish. The proliferation cells were detected in papilloma (blackdots area) by H\&E staining (Fig. 6b). Significantly, the much higher proportion of Ki-67 staining was detected in the epithelium of the yolk (Fig. 6c). Taking together, these data further support the contention that the constitutive activated MEK2 could induce proliferation in vitro and induce papilloma formation in transgenic zebrafish.

\section{Discussion}

In this study, we established a transgenic zebrafish model to explore the role of MEK2 in skin tumor formation. Constitutive MEK1 and MEK2 phosphorylated ERK1 and induced downstream MAPK signaling activation. Transient expression of constitutive MEK2 and MEK1 in the epidermis had induced skin papillary formation at $3 \mathrm{dpf}$. Sporadic skin papilloma formation was revealed in the epidermis of $\mathrm{Tg}(\mathrm{krt14}-\mathrm{MEK} 2 \mathrm{~S} 219 \mathrm{D}-\mathrm{GFP})$ transgenic zebrafish and had developed into skin tumors at $6 \mathrm{dpf}$. Treatment with U0126 could reduce skin tumor formation in $\operatorname{Tg}(\mathrm{krt14}-\mathrm{MEK} 2 \mathrm{~S} 219 \mathrm{D}-\mathrm{GFP})$ transgenic zebrafish.

The functions of MEK1 and MEK2 in the Ras/Raf/ Mek/Erk signaling pathway were well documented. During epidermal neoplasia formation, MEK2 and MEK1 showed mostly similar functions, but each had several differences. In zebrafish, the functions of MEK1 and

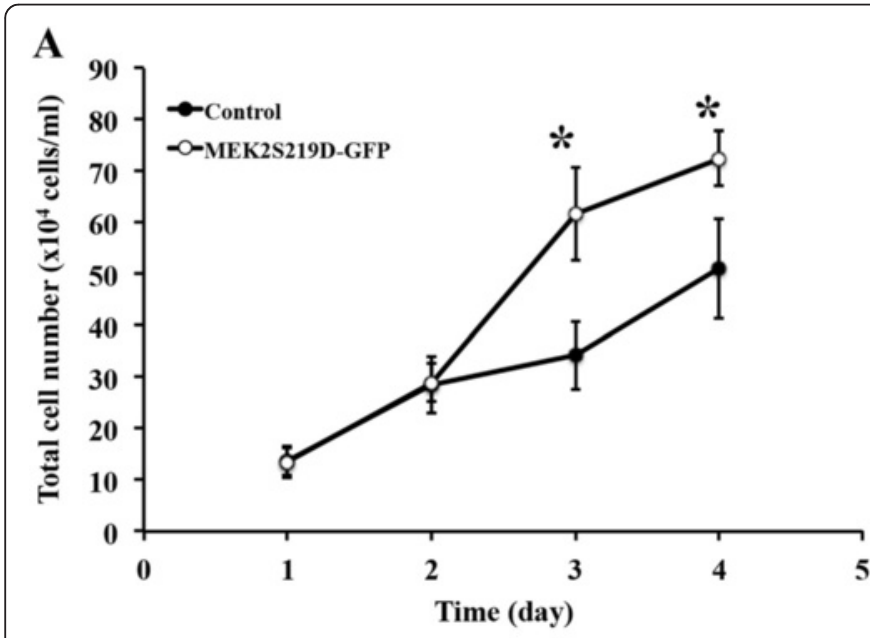

B
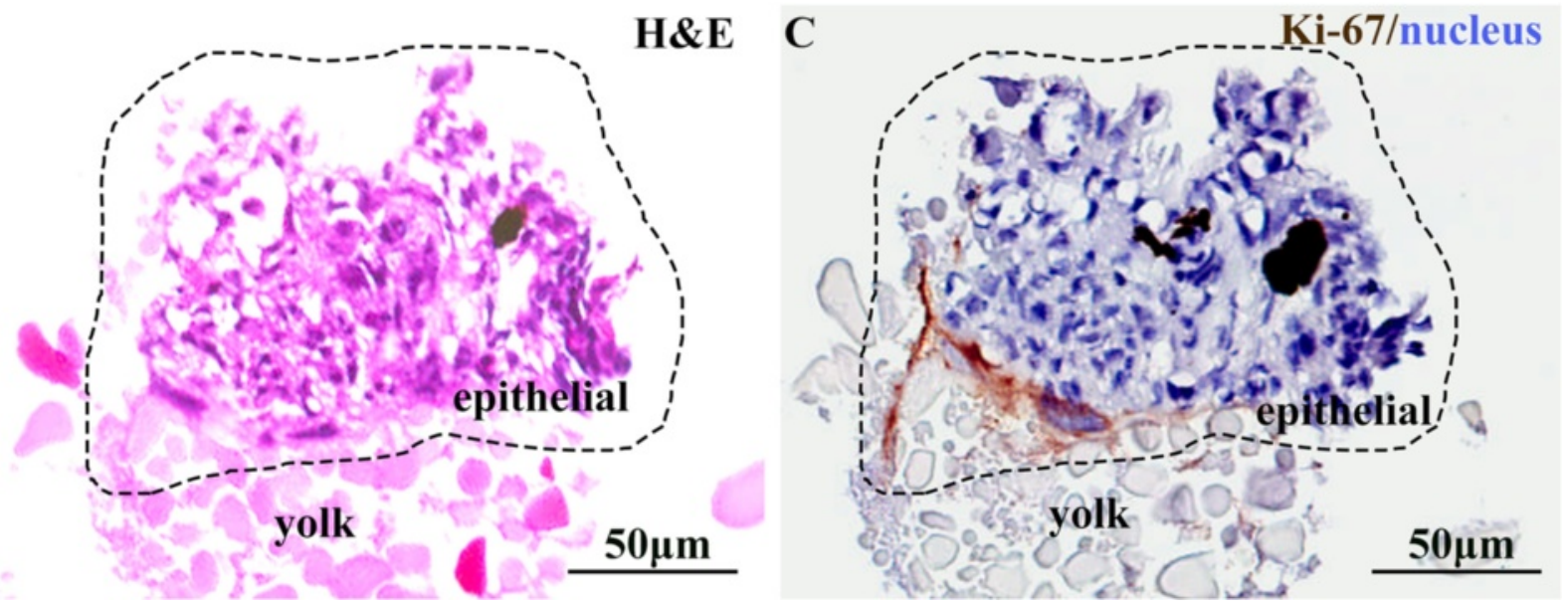

Fig. 6 Effect of MEK2S219D on cell proliferation was analyzed in COS-1 cells and in transgenic zebrafish. a The pcDNA3-MEK2S219D-GFP and pCDNA3-GFP (control) plasmids was transfected to COS-1 cells and evaluated the cell numbers by trypan blue assay from 1 to 4 days post-transfection. Results represent the mean of three independent determinations. * $p<0.05$. $\mathbf{b}$ H\&E-stained section in papilloma (black-dots area) of transgenic zebrafish. c Ki-67 IHC staining in papilloma (black-dots area) of transgenic zebrafish 
MEK2 have not yet been characterized. From a phylogenic analysis, we identified and isolated zebrafish mek1 and $m e k 2$ cDNAs. We also isolated the downstream erk1 cDNA to characterize the function of these two MEK proteins. An in vitro experiment showed that both MEK1 and MEK2 were able to phosphorylate downstream ERK1 by causing it to mutate to the constitutive active form. The ERK1 protein is then translocate from nuclei to the cytoplasm. We conclude that zebrafish MEK1 and MEK2 could activate downstream gene expression through Erk1 phosphorylation.

The role of MEK2 in skin tumor development is still being debated. MEK2 functions in mouse melanoma formation but does not contribute to skin tumor formation $[17,26,27]$. Those transgenic mice that contained chemically induced carcinomas or knockout mice were used to characterize the functions of MEK1 and MEK2. The krt14-MEK1 transgenic mice exhibited moderate hyperplasia with spontaneous skin tumor formation at 5 weeks. Downstream signaling is activated by ornithine decarboxylase (ODC) expression [7]. Surprisingly, skin tumors was sporadically formed just within 6 days in our $\operatorname{Tg}(\mathrm{krt14}-\mathrm{MEK} 2 \mathrm{~S} 219 \mathrm{D}-\mathrm{GFP})$ transgenic zebrafish. The zebrafish MEK2 was sufficient to induce skin tumor formation through ERK1 activation.

In previous reports, the function of MEK1 was important in skin tumor formation. In our results, transient expression of activated MEK1 was produced papillary formation in the epidermis. The skin papilloma was also revealed in activated MEK2 expression. Although we did not establish transgenic zebrafish by expression of activated MEK1, we suggest that MEK1 also potentially to induce skin papilloma formation. Moreover, transient expression of constitutive MEK2 in the epidermis induced papillary formation. These papillae were then excised from the epidermis and placed in the medium. Collection of this skin cells and cultured in L15 medium could be continuously maintained for more than 1 week. Analysis of these skin cells showed intact nuclei, tubulin structure, and cell shape (data not shown). We suggest that these skin cells were experiencing hyper-proliferation due to MEK2 activation. This observation may be relevant to psoriasis but needs further investigation.

Ras proto-oncogenes are central regulators activating intracellular Ras-Raf-MEK-ERK signaling pathway in tumorigenesis. The zebrafish is increasingly used as a vertebrate model for studying tumorigenesis and drug screening. A transgenic approach is generally used for establishing a transgenic zebrafish. The $\mathrm{Tg}$ (fabp10:EGFP$\mathrm{kras}^{\mathrm{v} 12}$ ) trangenic zebrafish specifically overexpressed high level of $\mathrm{kras}^{\mathrm{v12}}$ in liver and progressed to hyperplasia and malignant tumor [22, 23]. Furthermore, the transgenic zebrafish overexpressed the $\mathrm{BRAF}^{\mathrm{V} 600 \mathrm{E}}$-mutated gene activated under microphthalmia-associated transcription factor (mitf) promoter induced melanocyte proliferation [25]. The transgenic zebrafish also overexpressed kras $^{\mathrm{G} 12 \mathrm{~V}}$ gene by using a pancreas specific transcription factor 1a promoter expressed in the pancreases, which caused pancreatic neoplasia [6]. The overexpression of the kras ${ }^{\mathrm{G} 12 \mathrm{~V}}$ gene driven by cytokeratin 5 $(k r t 5)$ or glial fibrillary acidic protein promoter induced brain tumor [13]. Mutagenesis induced by a random insertion into the genome through a Tol2 transposon-mediated transgenic approach was used as new method for studying gene function during development $[14,21,24]$. A Tol2 transposon-mediated enhancer trap contains GFP without a promoter was used for establishing transgenic lines, but no phenotype was observed in 37 transgenic lines [14]. The aforementioned method driven by the mini keratin 8 promoter in the Tol2 transposon-mediated approach increased the transgenic efficiency to $16 \%$, but only $3.57 \%$ of genes were inserted into the coding region [24]. The Tol2 transposonmediated enhancer-trap using an hsp70 promoter-driven GFP was used to screen gene mutagenesis. The transgenic efficiency was increased to $70 \%$ but only $2.73 \%$ recessive mutations were identified [21]. To date, no gene mutation induced by Tol2 transposon-mediated enhancer trap has induced tumorigenesis. In our study, we established a Tol2 transposon-mediated $\mathrm{Tg}$ (Ka14:MEK2S219D-GFP) transgenic zebrafish by using a Ka14 promoter to constitutively activate MEK2S219D-GFP expression. Skip papilloma was observed at 3-6 dpf, and no other tumor type was observed in this transgenic line. We also established a $\mathrm{Tg}$ (Tol2:GFP) transgenic line by using Tol2 transposonmediated approach, which did not display skin papilloma. Although we could not complicatedly exclude the possibility of a random insertion to the oncogene upstream of MEK and induction of the skin papilloma formation, we suggested that the frequency was extremely rare.

In summary, we showed that MEK2 is sufficient to induce skin papilloma formation. In vitro and in vivo experiments showed that activated MEK2 in MAPK pathway promoted downstream signaling through ERK1 phosphorylation. This activation could be inhibited by U0126 and reduced skin papilloma formation in transgenic zebrafish. This $\mathrm{Tg}(\mathrm{krt14}-\mathrm{MEK} 2 \mathrm{~S} 219 \mathrm{D}-\mathrm{GFP})$ is the first established skin tumor model in zebrafish and could provide for anticancer drug screening to target the MAPK cascade.

\section{Conclusions}

Most cancer was associated with constitutive activation of ERK signaling, which was activated ERK1/2 by MEK1 and MEK2 upon activation of receptor tyrosine kinase. Our data supported that the constitutive activation of MEK2 was sufficiently induced tumor 
formation in $\operatorname{Tg}(\operatorname{krt14}$ :MEK2S219D-GFP) transgenic zebrafish. This transgenic zebrafish might be provided for anticancer drug screening.

\section{Competing interests}

The authors have no conflict of interest.

\section{Authors' contributions}

Chih-Ming Chou carried out the molecular genetic studies, participated in the sequence alignment. Yi-Chung Chen carried out the IHC experiemts. San Su performed the H\&E staining and IHC experiments. Gen-Der Chen carried out the molecular cloning of cDNAs. Kai-Yun Huang carried out the westen-blotting and immune-staining experiments. Huang-Wei Lien carried out the microinjection of embryos and establishment of transgenic zebrafish. Chang-Jen Huang and Chia-Hsiung Cheng designed, coordination and helped to draft the manuscript. All authors read and approved the final manuscript.

\section{Acknowledgments}

Taipei Medical University New Faculty Grant (TMU101-AE1-B22) and MOST (104-2311-B-038-001) supported this study. We thank the Zebrafish Core Laboratory of Taipei Medical University for help with the zebrafish experiments. We also thank the Department of Pathology of TMU for preparing H\&E and IHC stains.

\section{Author details}

'Department of Biochemistry and Molecular Cell Biology, School of Medicine, College of Medicine, Taipei Medical University, Taipei 110, Taiwan. ${ }^{2}$ Graduate Institute of Medical Sciences, College of Medicine, Taipei Medical University, Taipei 110, Taiwan. ${ }^{3}$ Institute of Biological Chemistry, Academia Sinica, 128 Academia Rd., Sec 2, Taipei 115, Taiwan. ${ }^{4}$ Department of Biochemistry, School of Medicine, College of Medicine, Taipei Medical University, 250, Wuxing Street, Taipei 11013, Taiwan.

Received: 21 April 2015 Accepted: 16 October 2015

Published online: 17 November 2015

\section{References}

1. Ahmad N, Gilliam AC, Katiyar SK, O'Brien TG, Mukhtar H. A definitive role of ornithine decarboxylase in photocarcinogenesis. Am J Pathol. 2001;159(3):885-92.

2. Bizub D, Wood AW, Skalka AM. Mutagenesis of the Ha-ras oncogene in mouse skin tumors induced by polycyclic aromatic hydrocarbons. Proc Natl Acad Sci U S A. 1986;83(16):6048-52.

3. Bos JL. ras oncogenes in human cancer: a review. Cancer Res. 1989;49(17):4682-9.

4. Castro P, Soares P, Gusmao L, Seruca R, Sobrinho-Simoes M. H-RAS 81 polymorphism is significantly associated with aneuploidy in follicular tumors of the thyroid. Oncogene. 2006;25(33):4620-7.

5. D'Cruz CM, Gunther EJ, Boxer RB, Hartman JL, Sintasath L, Moody SE, et al. C-MYC induces mammary tumorigenesis by means of a preferred pathway involving spontaneous Kras2 mutations. Nat Med. 2001;7(2):235-9.

6. Davison JM, Woo PS, Rhee JM, Leach SD. Characterization of kras-mediated pancreatic tumorigenesis in zebrafish. Methods Enzymol. 2008;438:391-417.

7. Feith DJ, Bol DK, Carboni JM, Lynch MJ, Sass-Kuhn S, Shoop PL, et al. Induction of ornithine decarboxylase activity is a necessary step for mitogen-activated protein kinase kinase-induced skin tumorigenesis. Cancer Res. 2005;65(2):572-8.

8. Fischer H, Eckhart L, Mildner M, Jaeger K, Buchberger M, Ghannadan M, et al. DNase1L2 Degrades Nuclear DNA during Corneocyte Formation. J Invest Dermatol. 2006;127(1):24-30

9. Fuchs $\mathrm{E}$, Cleveland DW. A structural scaffolding of intermediate filaments in health and disease. Science. 1998;279(5350):514-9.

10. Gong Z, Ju B, Wang X, He J, Wan H, Sudha PM, et al. Green fluorescent protein expression in germ-line transmitted transgenic zebrafish under a stratified epithelial promoter from keratin8. Dev Dyn. 2002;223(2):204-15.

11. Gopalbhai K, Meloche S. Repression of mitogen-activated protein kinases ERK1/ERK2 activity by a protein tyrosine phosphatase in rat fibroblasts transformed by upstream oncoproteins. J Cell Physiol. 1998;174(1):35-47.

12. lida M, Towatari M, Nakao A, lida H, Kiyoi H, Nakano Y, et al. Lack of constitutive activation of MAP kinase pathway in human acute myeloid leukemia cells with N-Ras mutation. Leukemia. 1999;13(4):585-9.
13. Ju B, Chen W, Orr BA, Spitsbergen JM, Jia S, Eden CJ, et al. Oncogenic KRAS promotes malignant brain tumors in zebrafish. Mol Cancer. 2015;14(1):18.

14. Kawakami K, Takeda H, Kawakami N, Kobayashi M, Matsuda N, Mishina M. A transposon-mediated gene trap approach identifies developmentally regulated genes in zebrafish. Dev Cell. 2004;7(1):133-44.

15. Khosravi-Far R, Der CJ. The Ras signal transduction pathway. Cancer Metastasis Rev. 1994;13(1):67-89.

16. Krushna PB, Akimenko MA, Ekker M. Independent expansion of the keratin gene family in teleostean fish and mammals: an insight from phylogenetic analysis and radiation hybrid mapping of keratin genes in zebrafish. Gene. 2006;368:37-45.

17. Lee CS, Dykema KJ, Hawkins DM, Cherba DM, Webb CP, Furge KA, et al. MEK2 is sufficient but not necessary for proliferation and anchorage-independent growth of SK-MEL-28 melanoma cells. PLoS One. 2011;6(2), e17165.

18. Lenormand P, Sardet C, Pages G, L'Allemain G, Brunet A, Pouyssegur J. Growth factors induce nuclear translocation of MAP kinases (p42mapk and p44mapk) but not of their activator MAP kinase kinase (p45mapkk) in fibroblasts. J Cell Biol. 1993;122(5):1079-88.

19. McKillop IH, Schmidt CM, Cahill PA, Sitzmann JV. Altered expression of mitogen-activated protein kinases in a rat model of experimental hepatocellular carcinoma. Hepatology. 1997;26(6):1484-91.

20. Megosh L, Halpern M, Farkash E, O'Brien TG. Analysis of ras gene mutational spectra in epidermal papillomas from K6/ODC transgenic mice. Mol Carcinog. 1998;22(3):145-9.

21. Nagayoshi S, Hayashi E, Abe G, Osato N, Asakawa K, Urasaki A, et al. Insertional mutagenesis by the Tol2 transposon-mediated enhancer trap approach generated mutations in two developmental genes: tcf7 and synembryn-like. Development. 2008;135(1):159-69.

22. Nguyen AT, Emelyanov A, Koh CHV, Spitsbergen JM, Lam SH, Mathavan S, et al. A high level of liver-specific expression of oncogenic KrasV12 drives robust liver tumorigenesis in transgenic zebrafish. Dis Model Mech. 2011;4(6):801-13.

23. Nguyen AT, Emelyanov A, Koh CHV, Spitsbergen JM, Parinov S, Gong Z. An inducible krasV12 transgenic zebrafish model for liver tumorigenesis and chemical drug screening. Dis Model Mech. 2011;5(1):63-72.

24. Parinov S, Kondrichin I, Korzh V, Emelyanov A. Tol2transposon-mediated enhancer trap to identify developmentally regulated zebrafish genes in vivo. Dev Dyn. 2004;231(2):449-59.

25. Patton EE, Widlund HR, Kutok JL, Kopani KR, Amatruda JF, Murphey RD, et al. BRAF mutations Are sufficient to promote nevi formation and cooperate with p53 in the genesis of melanoma. Curr Biol. 2005;15(3):249-54.

26. Scholl FA, Dumesic PA, Barragan DI, Harada K, Bissonauth V, Charron J, et al. Mek1/2 MAPK kinases are essential for mammalian development, homeostasis, and Raf-induced hyperplasia. Dev Cell. 2007;12(4):615-29.

27. Scholl FA, Dumesic PA, Barragan DI, Harada K, Charron J, Khavari PA. Selective role for Mek1 but not Mek2 in the induction of epidermal neoplasia. Cancer Res. 2009;69(9):3772-8.

28. Tan X, Egami H, Kamohara H, Ishikawa S, Kurizaki T, Yoshida N, et al. Involvement of the mitogen-activated protein kinase kinase 2 in the induction of cell dissociation in pancreatic cancer. Int J Oncol. 2004;24(1):65-73.

29. Wang YH, Chen YH, Wu TN, Lin YJ, Tsai HJ. A keratin 18 transgenic zebrafish $\operatorname{Tg}(\mathrm{k} 18(2.9): \mathrm{RFP})$ treated with inorganic arsenite reveals visible overproliferation of epithelial cells. Toxicol Lett. 2006;163(3):191-7.

30. Westerfield M. The zebrafish book. 3rd ed. Eugene: University of Oregon Press; 1995.

\section{Submit your next manuscript to BioMed Central and take full advantage of:}

- Convenient online submission

- Thorough peer review

- No space constraints or color figure charges

- Immediate publication on acceptance

- Inclusion in PubMed, CAS, Scopus and Google Scholar

- Research which is freely available for redistribution 